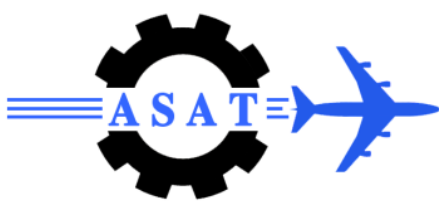

\title{
An Experimental Testing of an UAV Composite Wing Structure
}

\author{
Tariq H. Elsonni ${ }^{*}$ and M.M. Elnomrossy ${ }^{\dagger}$
}

\begin{abstract}
The wing of an UAV plays an important role in the vehicle behavior. In order to understand its main influences, a dedicated test rig was designed and realized to load the composite wing in the bending configuration. This study describes the details of a numerical and an experimental investigation done with the aim to identify the stiffness characteristics of a composite wing structure of an Unmanned Aerial Vehicle (UAV).
\end{abstract}

The wing was divided into discrete span-wise stations. Wooden bolted clamps were placed along the upper and lower surfaces of the wing at each station, to support the applied load and the displacement transducers. The wing was suspended and secured horizontally (at the wingfuselage connection base) in a rigid test stand in an orientation similar to the familiar cantilever beam. A single weight was applied individually, at each station to stress the wing in elastic yield, as in real conditions. Displacement transducers were installed to measure the vertical displacements of the entire wing, and monitor any motion of the overall airframe. During the measurement procedures (loading and unloading) were conducted on the specified stations, and repeated 10 times, the mean value of the measurements was obtained.

A detailed numerical model of the composite wing was developed using of the finite element method in order to reproduce the experimental experiences. Numerical and experimental results were compared to validate the model of the composite wing. All the obtained parameters are mainly being used in a development process of UAV behavior.

Keywords: UAV, wing, composite, test rig, stiffness test, F.E.M, flexibility

\section{Nomenclature}

E Young's modules

F Force

$G \quad$ Modulus of rigidity

K Total stiffness

$K \quad$ Stiffness matrix coefficient

\section{Abbreviations}

FEM Finite Element Method

UAV Unmanned Aerial Vehicle

\footnotetext{
Assistant professor, Department of Aeronautical Engineering, Karary University, Khartoum, Sudan.1nt273@yahoo.com.

$\dagger$ Professor, Production, Energy and Automatic Control Department, French University in Egypt, (Former President of Aerospace Research Center, Arab Organization of Industrialization), Egypt, AIAA member, elnomrossy@gmail.com .
} 


\section{Introduction}

Experimental investigation is an essential and indispensable step in developing and validating the design of UAV systems. Development of UAVs technology is of interest because it allows for adjustments to be made to the vehicle before actual flight, thus enlarging the flight envelope for the vehicle. Nevertheless the development process is a difficult procedure, which involves testing through simulation at first, and then actual experimentation on real vehicles. As simulation cannot accurately represent the exact real flight conditions and the dangers involved in them, this study and the resulting tests described herein are primarily motivated by the desire to conceive, develop and fill the gap between numerical runs and experimental ground and flight tests.

Simulations provide a useful development tool but the focus here is to describe the developed test-bed to demonstrate these control approaches in actual flight.

\section{Stiffness Testing}

Experimental testing was performed on a composite wing to determine its response to externally applied static loads at various wing stations. The methodology is presented starting with laboratory testing of the wing to determine its flexibility, and ending with the calculation of the wing stiffness matrix.

\section{Test Objectives}

- To measure the flexibility of the composite wing.

- To determine the stiffness matrix of the wing.

- Establish a baseline stiffness signature for the wing before model modification.

- Validate the repeatability of the test results.

- Make comparisons with FEM predictions

\section{Test Setup}

\section{Description of the test article}

For this experiment, the whole wing with the baseline geometry [1, 2], given in Table 1, was divided into 8 spanwise stations (4 on each side of the wing), as shown in Fig. 1. Wood clamps were attached to support the applied load and the displacement measuring transducers. Clamps were placed along the upper and lower surfaces of the wing at each station and bolted together, Fig. 2.

The wing was suspended and secured horizontally (at the wing-fuselage connection base) in a rigid test stand in an orientation similar to the familiar cantilever beam as shown in Fig. 3 . The rigid test stand was fixed to a concrete floor as shown in Fig. 4.

Displacement transducers, Fig. 5, were installed to measure the vertical displacements of the wing at different stations, and monitor any motion of the overall airframe. Measurements were conducted on numbers of nodes specified on each spanwise station; the whole wing has a total of (28)-twenty eight nodes, (fourteen on each side of the wing), see Fig. 1.

\section{Test equipment}

Stiffness tests were conducted using the HBM Data Acquisition System. The complete system consists of four racks, containing 128 DAQ-channels each, Fig. 6. Each rack is equipped with an Ethernet computer interface. All racks are linked together with a synchronization cable. The data communication is done via Ethernet. The system consists of: 

a) Server computer
b) 4-MGCplus units, 3 for strain measurements, and one for displacement measurements, Fig. 7.
c) Each MGCplus contains 16 slots, each slot contains 8 channels.
d) ML801 Multi-Channel Amplifier
e) AP 815 Connection Board.
f) Power Supply for 128 LVDT FTH7311.
g) DAQ Cabinet.
h) MD Server software package.

It should be noted that the system is designed to operate within environmental conditions of (10-40) ${ }^{\circ} \mathrm{C}$, and (0-95) \% humidity.

\section{Test procedures}

The load was applied as a concentrated force at each node. All testing was performed at the Aerospace Research Center Laboratories (static and fatigue laboratory). Data were acquired and displayed by the laboratory data acquisition and control system.

The test procedure can be summarized as follows:
a) The data system was zeroed. time), and then operator waited for 3-5 minutes till the structure became relaxed. This step is shown in Figs. 8. and 9.
b) A point load of $6.5 \mathrm{~kg}$ was hung at one node (at each side of the wing and at the same
c) The corresponding readings were recorded.
d) The data system was zeroed again.
e) The weight was increased from 11.5 to $51.5 \mathrm{~kg}$ gradually, and steps 3 and 4 were repeated for each weight.
f) The weight was decreased gradually from 51.5 to $6.5 \mathrm{~kg}$ until zero, and then steps 3 and 4 were repeated respectively for each weight.

The measuring procedure (loading and unloading) was repeated ten (10) - times and the mean values of the measurements were calculated. Ten measurements were performed with point loads of varying magnitude, starting with load increment condition, and then flipped until zero unloading condition (Fifteen loading steps).

During testing, the applied loads had equal magnitudes and applied in the same direction on each side of the wing. Each test began with a zero loading condition, and then a smooth sweep was performed for the remaining loads. The weights were attached to the wing as shown in Fig. 10.

The unit-load method is useful for determining the displacement at number of nodes in a structure. In designing air-vehicles it is usually necessary to determine the displacement at a large number of nodes in the structure for many different load conditions. As the flexibility matrix relates nodal displacements to nodal forces, the general displacements and nodal forces are related by the following equation, [3]:

$$
\delta_{i j}=\frac{\delta_{i}}{F_{j}}
$$

where $\delta_{i j}$ the flexibility is influence coefficient, and $\delta_{i}$ is the displacement at node $i$ due to a unit load $F_{j}$. 
Considering the physical characteristics of the structure, the flexibility matrix is produced from the superposition of nodes solution. Applying a unit load vertically and individually at only one node on each side of the wing (opposite nodes must be selected) and at the same time while keeping the rest of the nodes unloaded; the magnitude of the displacement at each node is then measured using displacement transducers. The procedure is repeated for the remaining nodes.

Using equation (1), the flexibility is evaluated in a matrix form for 14-degrees of freedom as:

$$
[F]=\left[\delta_{i j}\right]=\left[\begin{array}{ccccc}
\delta_{1,1} & \delta_{1,2} & \cdot & \cdot & \delta_{1,14} \\
\delta_{2,1} & \delta_{2,2} & \cdot & \cdot & \cdot \\
\cdot & \cdot & \cdot & \cdot & \cdot \\
\cdot & \cdot & \cdot & \cdot & \cdot \\
\delta_{14,1} & \cdot & \cdot & \cdot & \delta_{14,14}
\end{array}\right]
$$

with $\delta_{i j}=\delta_{j i}$

The stiffness matrix coefficients are $K_{i j}$ determined as seen in equation (3). The formulation includes; measuring the displacements on the wing, computing the flexibility matrix, and then building up the stiffness matrix.

By following these steps, and based on the nodal force-displacement relation, one can write the stiffness matrix coefficients in the form:

$$
K_{i j}=\frac{F_{i}}{\delta_{j}}
$$

The flexibility matrix given in matrix (2) is important to form the stiffness matrix as given in matrix (4) as follows:

$$
[K]=\left[K_{i j}\right]=\left[\begin{array}{ccccc}
K_{1,1} & K_{1,2} & \cdot & \cdot & K_{1,14} \\
K_{2,1} & K_{2,2} & \cdot & \cdot & \cdot \\
\cdot & \cdot & \cdot & \cdot & \cdot \\
\cdot & \cdot & \cdot & \cdot & \cdot \\
K_{14,1} & \cdot & \cdot & \cdot & K_{14,14}
\end{array}\right]
$$

\section{Results of Experimental Test and Statistical Analysis}

The feasibility and effectiveness of the applied method in evaluating the stiffness of the composite wing was examined by measuring the displacements at specified locations on the top of the wing and following the above mentioned procedures.

To examine the behavior of the wing subjected to unit loads, the displacement was measured at each of the fourteen nodes along the span due to a unit load at one point, (see Fig. 1. for nodes location). For illustration purpose, only one node has been selected, hence Fig. 11 shows measurements taken at the tip of the leading edge (node\#1 as in Fig. 1) Examining this figure, the following points are noteworthy:

The measured values at each point are validated individually for each loading level.

The validated values at each point and for different loading levels show the linearity of measurements (within the range of the displacement transducers and also material characteristics). 
In this figure the best-fit line, including $95 \%$ confidence interval bands is indicated. This shows the relationship between the applied load and the measured displacements. The displacements values tend to be around the best-fit line everywhere. The lower and upper bounds for $95 \%$ confidence level assigned to the mean were also shown in each figure.

Figure 12 shows the response of the wing structure during loading and unloading conditions. The data of points corresponding to zero loading show deformation after removing the largest loading, again showing hysteresis in the wing response. As displacements were measured at specified nodes on each side of the wing, data were processed and the following results give further examination of the composite wing behavior:

As test was being performed, a noticeable twist was evident at the wing tip (nodes (1-2)) as seen in Fig. 13. It is seen that the vertical displacement of the leading edge due to loading is less than that of the trailing edge. It is evident from this figure that the trailing edge of the wing is less stiff than the leading edge.

Displacement along wing root at nodes $(10,11,12,13$, and 14) is shown in Fig. 14. From this figure, it is seen that displacement varies linearly with the applied load.

Displacement along wing root at nodes $(10,11,12,13$, and 14) in case of unloading is shown in Fig. 15.

Displacement along wing root at nodes $(10,11,12,13$, and 14) in case of max loading is shown in Fig. 16.

Displacement along wing trailing edge at nodes $(2,5,9$, and 14) is given in Fig. 17.

In the context of this data, the flexibility matrix is generated as follows:

Nodal displacements were evaluated on the entire wing under unit loads. The measured displacements on both sides of the wing were found symmetric; hence only one half of the wing was considered, (measurements were conducted on 14 nodes). The results are tabulated in Table 2.

Measurements were recorded for 15 loading conditions and repeated for 10-times on each of the 14 nodes.

Statistical analysis was performed on the data using linear regression. When the measured data was validated, equation (1) was applied to build up the flexibility matrix (2).

Using this result, the stiffness matrix was constructed by inverting the flexibility matrix. The stiffness matrix exhibits the property of diagonal symmetry, hence the following 14-by-14 stiffness matrix, listed to 4 decimal places was obtained as presented in matrix (4). The spatial distribution of stiffness contributes to the instantaneous shape of the wing under load, and hence is crucial to understanding the effect of structural design of the composite wing on airvehicle performance.

\section{Finite Element Modeling and Results}

As in the standard procedure for building MSC.Patran models, the wing geometry is to be built first, and then a finite element mesh is to be constructed on this geometry. The geometry will proceed from creation of points to lines to surfaces for this model. Next, the finite 
element method requires the knowledge of the physical parameters of the desired structure to relate a force input to a displaced output. The whole wing was built as a plate. The finite element model for this work utilized the general-purpose element, QUAD4 to model the composite wing. These families of elements are the most commonly used 2-D elements in the MSC/NASTRAN element library [4, 5].

The internal structure of the real wing is composed of upper and lower skins; injected foam between skins, and one spar with rectangular cross- section made of wood and located at wing trailing edge, Fig. 18. All layers are modeled as a composite laminate, (stacked plies used to form the skin). The foam is modeled as a brick element, and a one-dimensional element was used to represent the spar structural member $[5,6]$. When meshing the wing, the areas having different staking are made into separate elements and each element gets its suitable property. Several problems arise due to the irregular shape of the airfoil and due to the irregular variation in foam thickness between the upper and lower skins. So the wing was divided into sections and the foam average thickness was calculated for each section. When building a composite property for an element, the foam thickness inserted as an average thickness in the wing zone.

Figure 19 illustrates the composite wing finite element model built-up in MSC.NASTRAN, while the properties of the actual materials are presented in Table 3.

For validation purposes besides exploring the inherent stiffness of the composite wing, the model was created to give high resemblance of the real wing structure and, mimicking the displacements range measured in the real wing. The displacements are calculated and tabulated as in Table 4.

When correlating the displacements measured experimentally with those calculated by the finite element, the correlation reveals a noticeable dissimilarity between the two results. This dissimilarity is to be expected due to the distinct nature of the analysis methods used. The finite element analysis is an analytical technique, and therefore yields the matched displacements exhibited above. Conversely, the stiffness test is an experimental technique. Thus, the results are not the matching displacements predicted by analytical and experimental techniques. In other words; the results obtained by finite element model, Table 4 represent the THEORETICAL ROUTE for the analysis. We proceed from spatial model through to a response analysis as seen in Fig. 20. The figure illustrates the three phases through which a typical theoretical analysis progresses.

At first the SPATIAL MODEL describes the structure's physical characteristics. Secondly the MODAL MODEL describes the structure's behavior. Finally the RESPONSE MODEL describes the analysis of the structure's response.

The result obtained by the experimental work, Table 2, represents the reverse direction that the analysis undertakes. From a description of the response properties, the modal properties can be deduced and, in the limit, leading to the spatial properties. This is the EXPERIMENTAL ROUTE to the analysis, and is shown in Fig. 21.

It must be noted that an experimentally based technology demands richness of theoretical methods that significantly outstrips the corresponding material found in a theoretically based study of the same subject. This is simply because in the experimental field we must be prepared to explain and to interpret the most general of circumstances. The luxury of being 
able to dictate the conditions (or assumptions) at the outset of study, which we want to do in theoretical analyses - is not one that can generally be extended to the experimentalist, and so must be armed with the most general of models.

The preceding theory has been concerned with complete models (the one which is fully defined by its description). While this is a valid approach for theoretical study, it is less generally applicable for experimentally based investigations. Because of this limitation, the concept of a 'reduced' or 'incomplete model' (the one in which some information is reduced or eliminated) was defined.

Based on these facts, it is clear that both approaches (theoretical route and experimental route) represent incomplete models of the physical system.

\section{Conclusion}

An experimental test to quantify the wing flexibility and stiffness matrix was performed by mounting the wing to a rigid test stand. Equal magnitude loads ranging from $6.5 \mathrm{~kg}$ to $51.5 \mathrm{~kg}$ were applied vertically and downward at only one node on each side of the wing (opposite nodes were selected), while the rest nodes were kept unloaded. Loadings were conducted by applying hanging weights to the node. Displacement transducers were used to measure the actual displacement at each node on the entire wing due to a unit applied load.

The stiffness test results showed slightly softening behavior, with increasingly larger displacements occurring as the load was increased. The softening trend is more pronounced for the lowest load of $6.5 \mathrm{~kg}$. Still for all other loads, a linear approximation of the incremental loading response is reasonable. Displacement values corresponding to the zeroloading and unloading levels were recorded.

The primary objectives of these experimental tests were to characterize the wing behavior before flight, and provide a before-and-after measurement of the stiffness. The test results could be used to modify the finite-element model (FEM), and for further aeroelasticity predictions and optimization developments. Displacements from these tests were computed to evaluate the accuracy of the model stiffness, and determine whether modifications to the model were necessary.

Data gathered in this experiment produced fairly accurate results, and showed good repeatability between the tests.

As predicted by the theory, the data using different weights to determine stiffness were capable of fitting to straight lines.

\section{References}

[1] "Preliminary sizing of SAHM-UAV", Aerospace Research Center, 1997.

[2] "Configuration design of SAHM-UAV", Aerospace Research Center, 1997.

[3] “MSC.NASTRAN", Reference manual, 2005.

[4] "MSC.PATRAN, Reference manual, Part 5: Analysis application, 2005.

[6] "MSC.NASTRAN, Release guide, 2005 
Table 1 Wing Geometry [1, 2]

\begin{tabular}{l|l}
\hline \multicolumn{1}{c||}{ Parameter } & \multicolumn{1}{|c}{ Value } \\
\hline Span [ft] & 7.1 \\
\hline Quarter chord sweep angle [deg] & 30 \\
\hline Root chord [ft]-(NACA-0009-airfoil) & 4.92 \\
\hline Tip chord [ft]- (NACA-0009-airfoil) & 1.5 \\
\hline Area [ft2] & 22.6 \\
\hline \hline
\end{tabular}

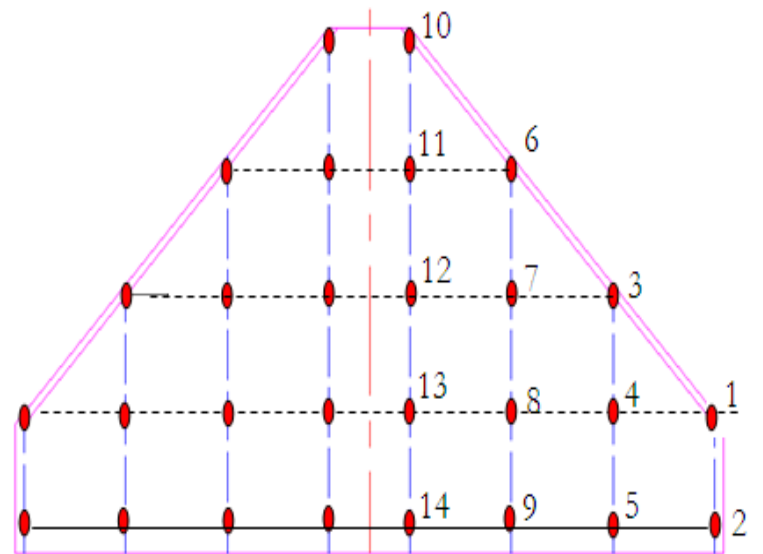

Fig. 1 Wing stations \& nodes arrangement

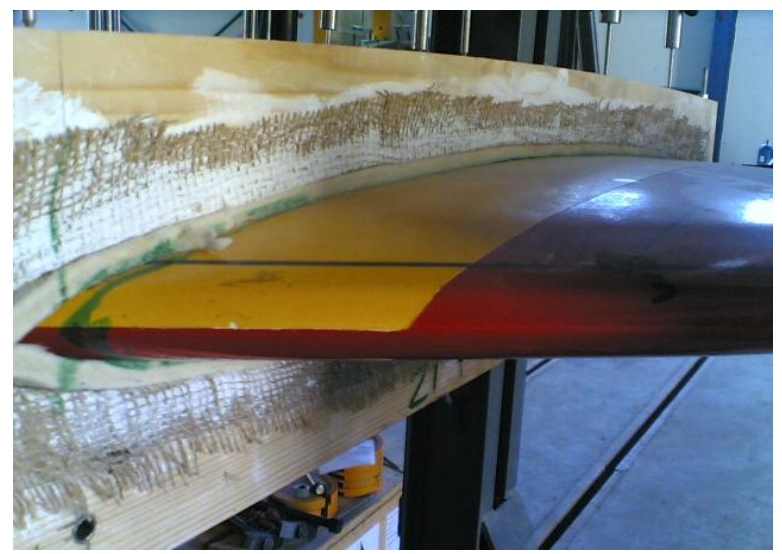

Fig. 3 Wing attached to a rigid test stand

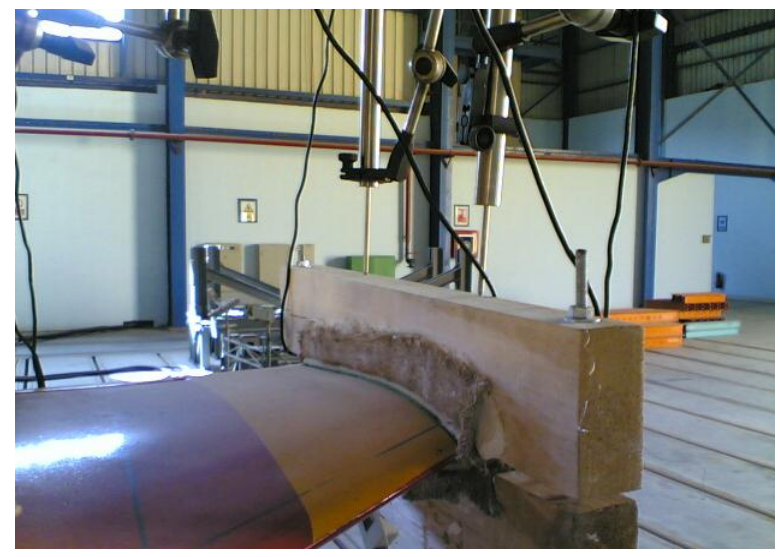

Fig. 5 Displacement transducer

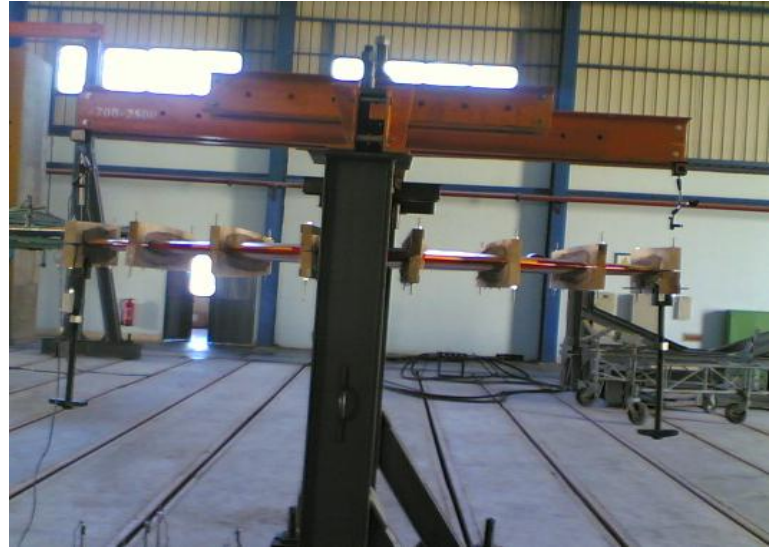

Fig. 2 Clamps along wing

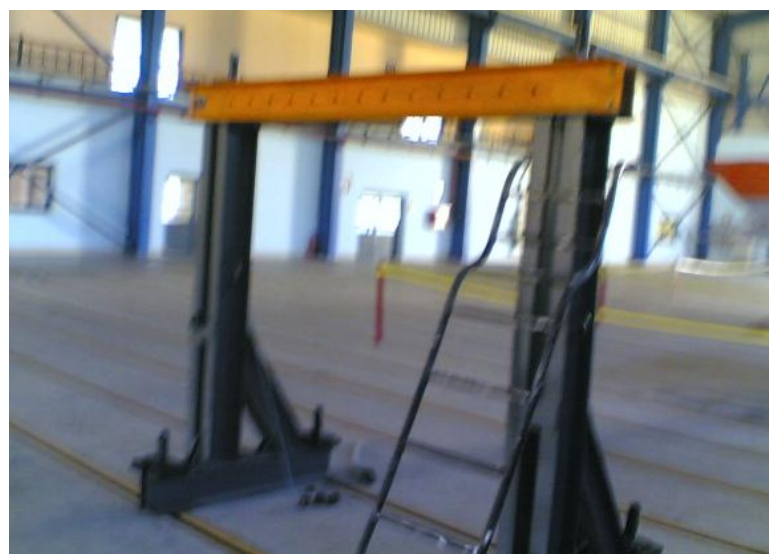

Fig. 4 Rigid stand fixed to concrete

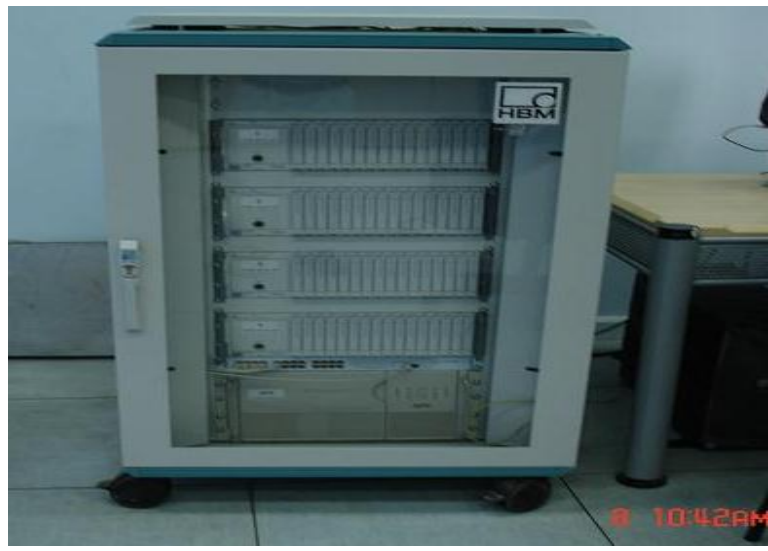

Fig. 6 HBM Data acquisition system 


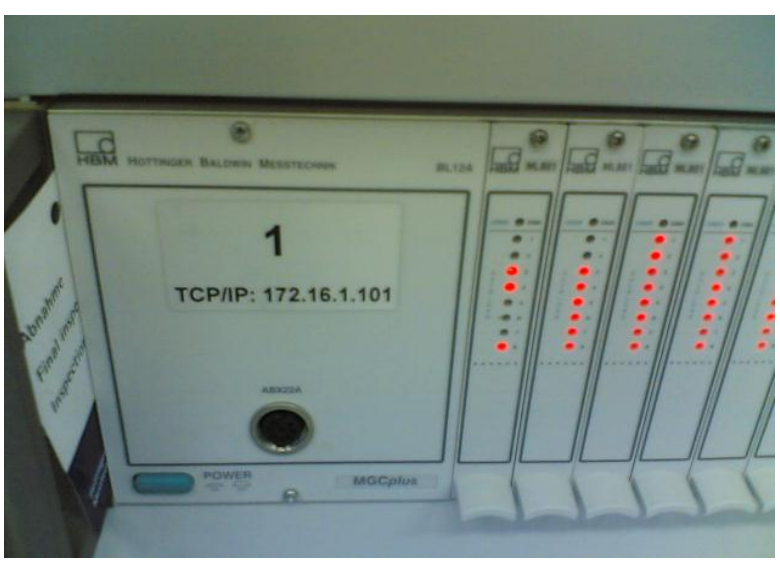

Fig. 7 MGCplus unit

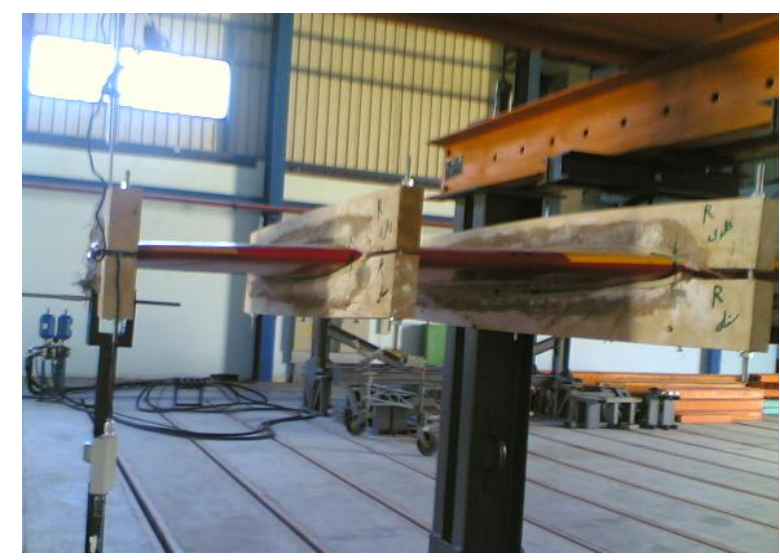

Fig. 9 Load applied to other side of the wing

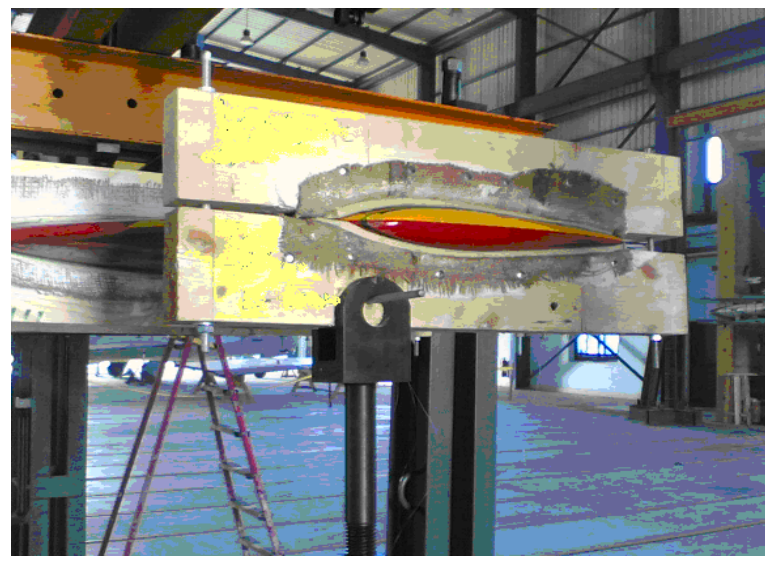

Fig. 8 Load applied to one side of the wing

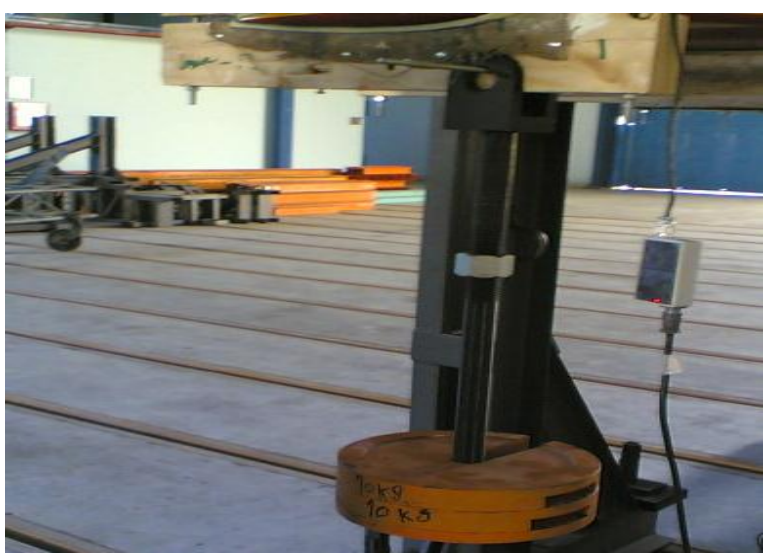

Fig. 10 Weights attached to wing

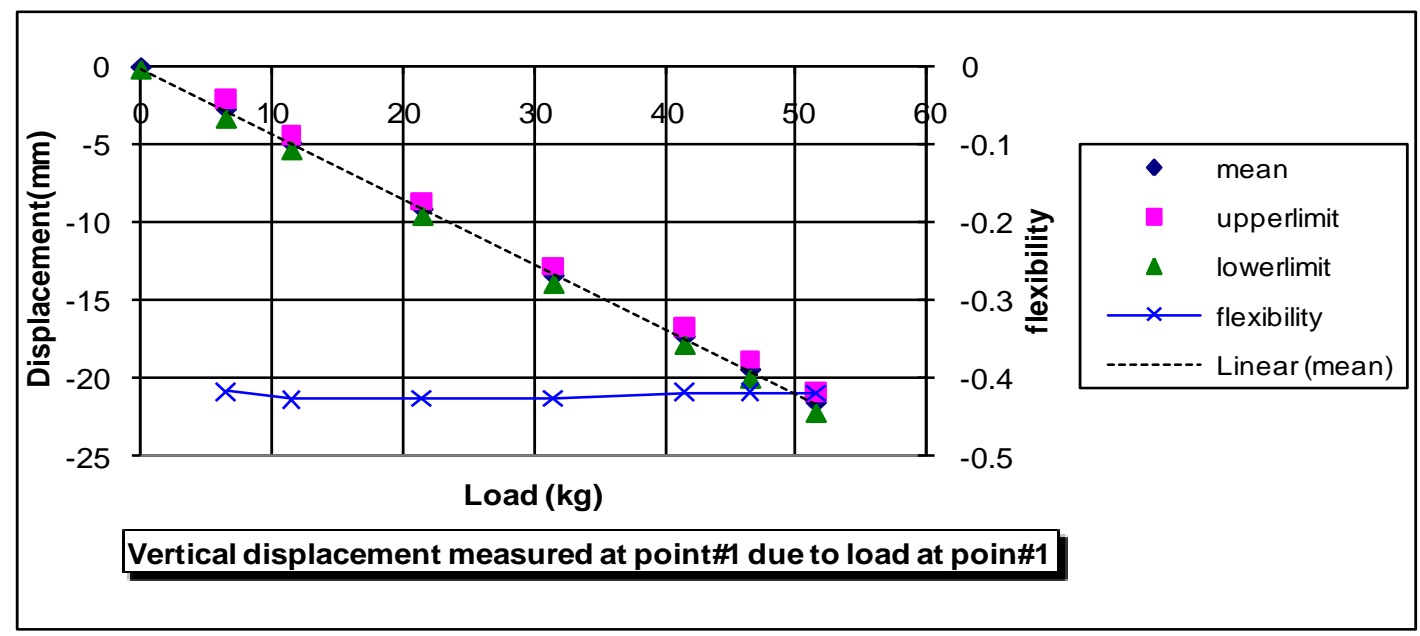

Fig. 11 Vertical displacement measured at point \#1 due to load at point \#1 


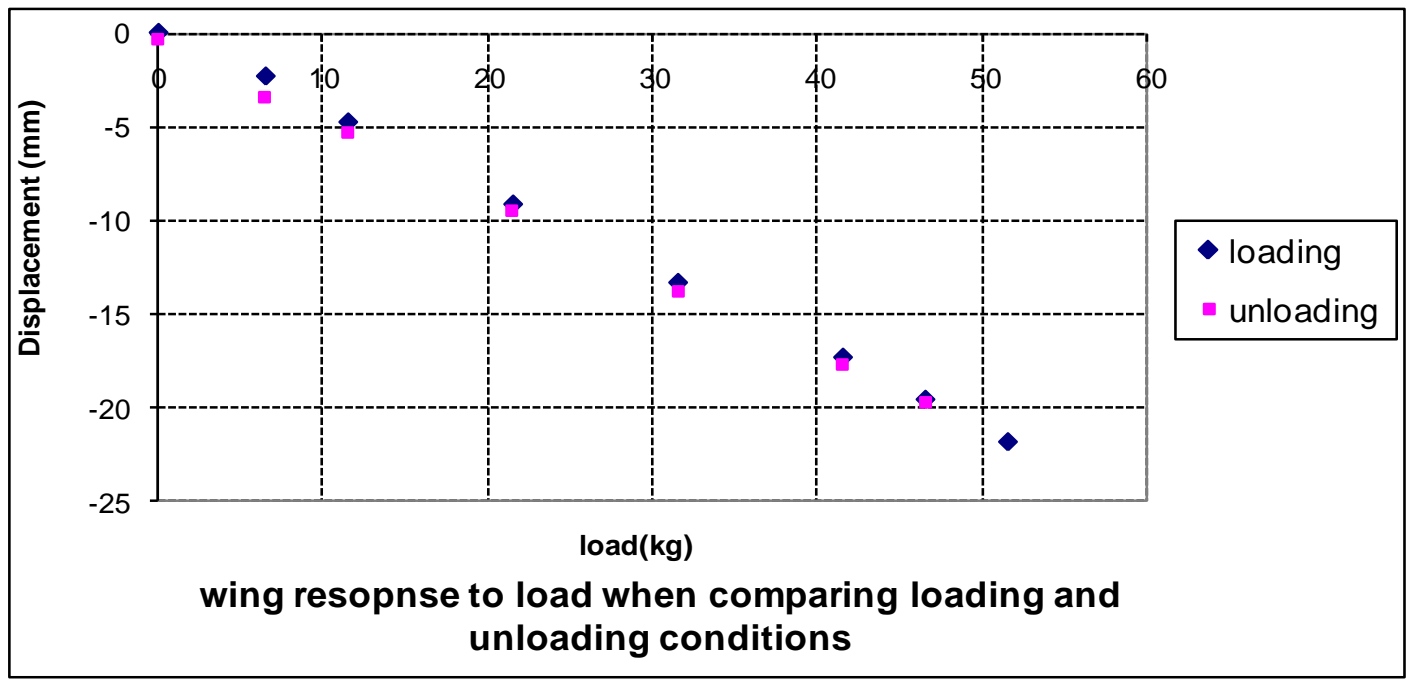

Fig. 12 Response of wing structure during loading and unloading

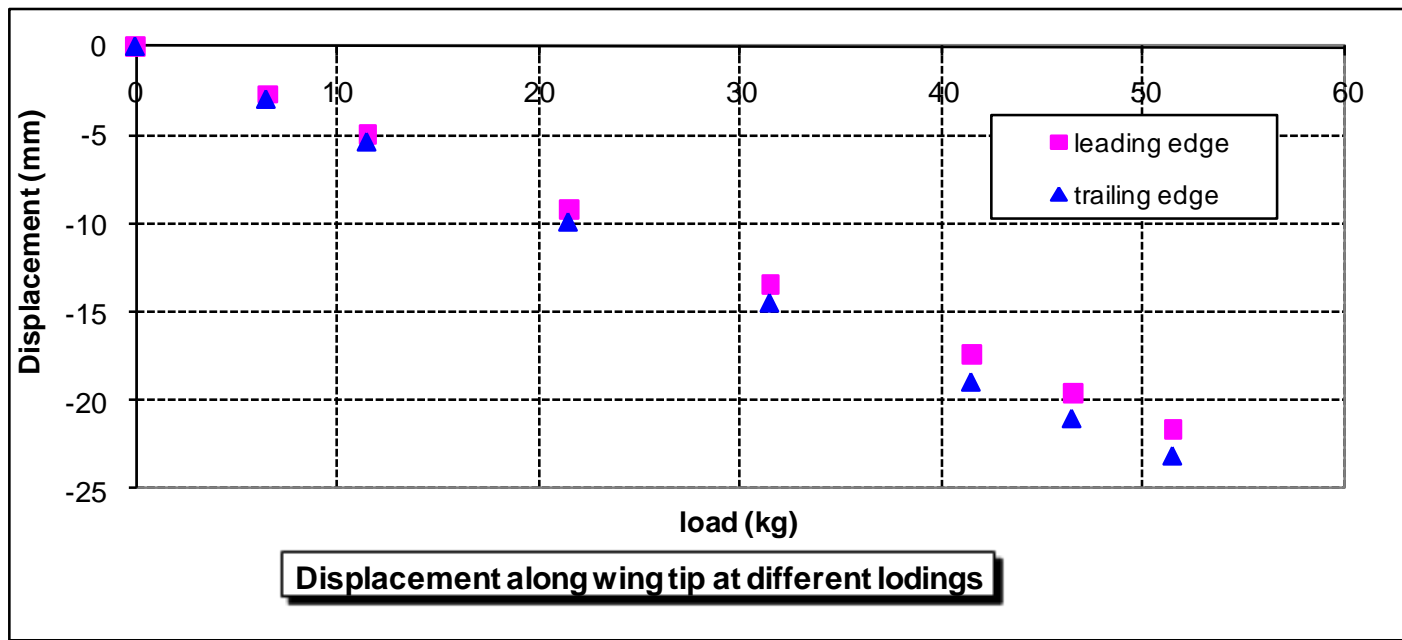

Fig. 13 Displacement along wing tip at nodes (1-2)

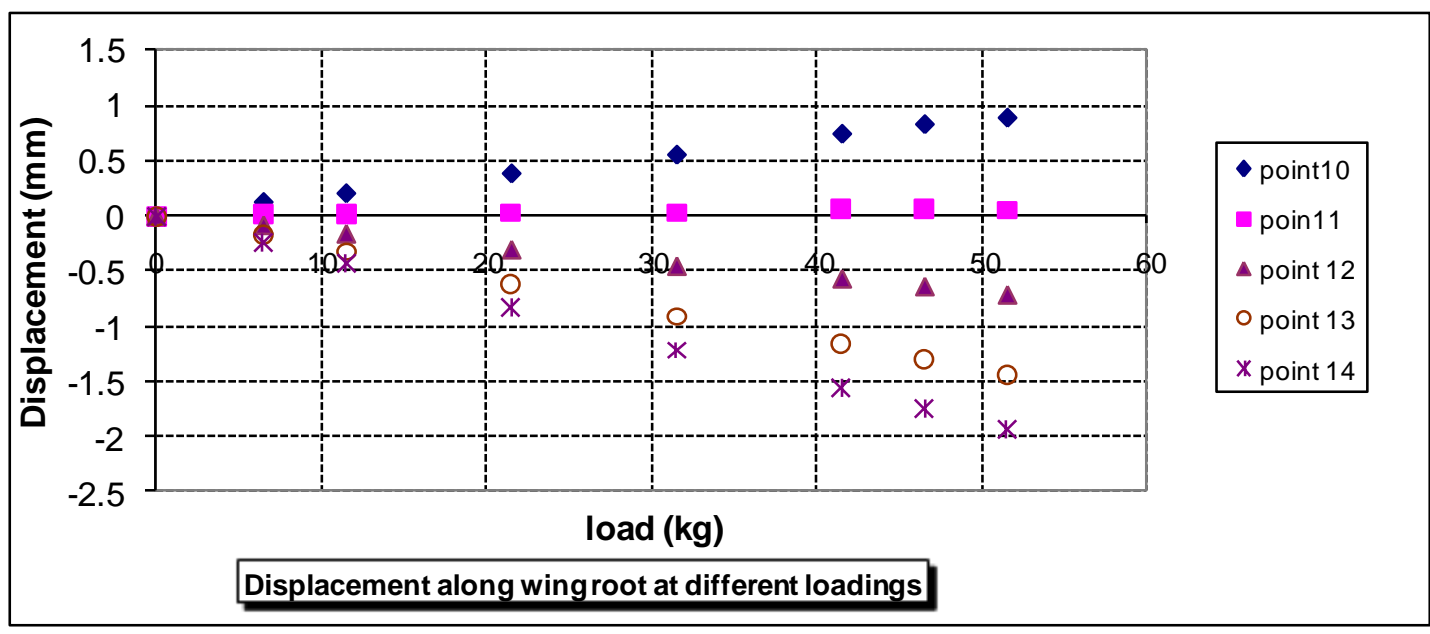

Fig. 14 Displacement along the root at nodes $(10,11,12,13$, and 14) 


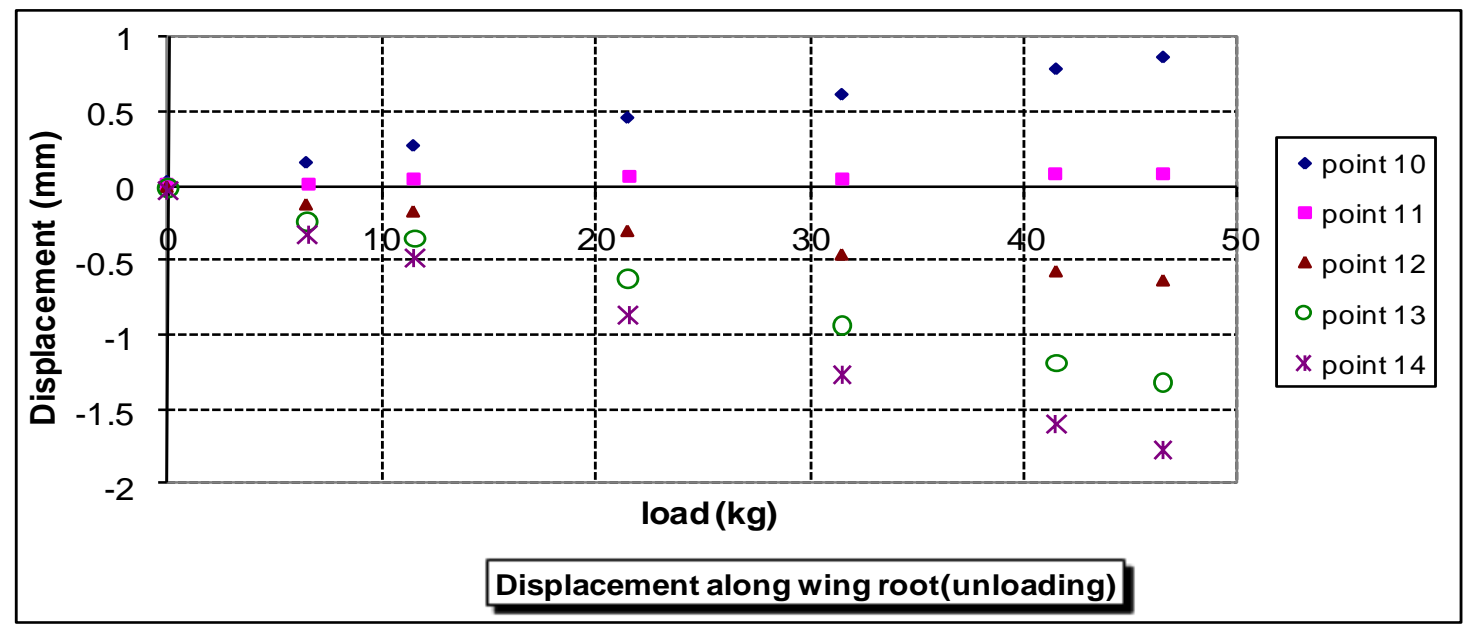

Fig. 15 Displacement along the root (nodes 10, 11, 12, 13, and 14-unloading)

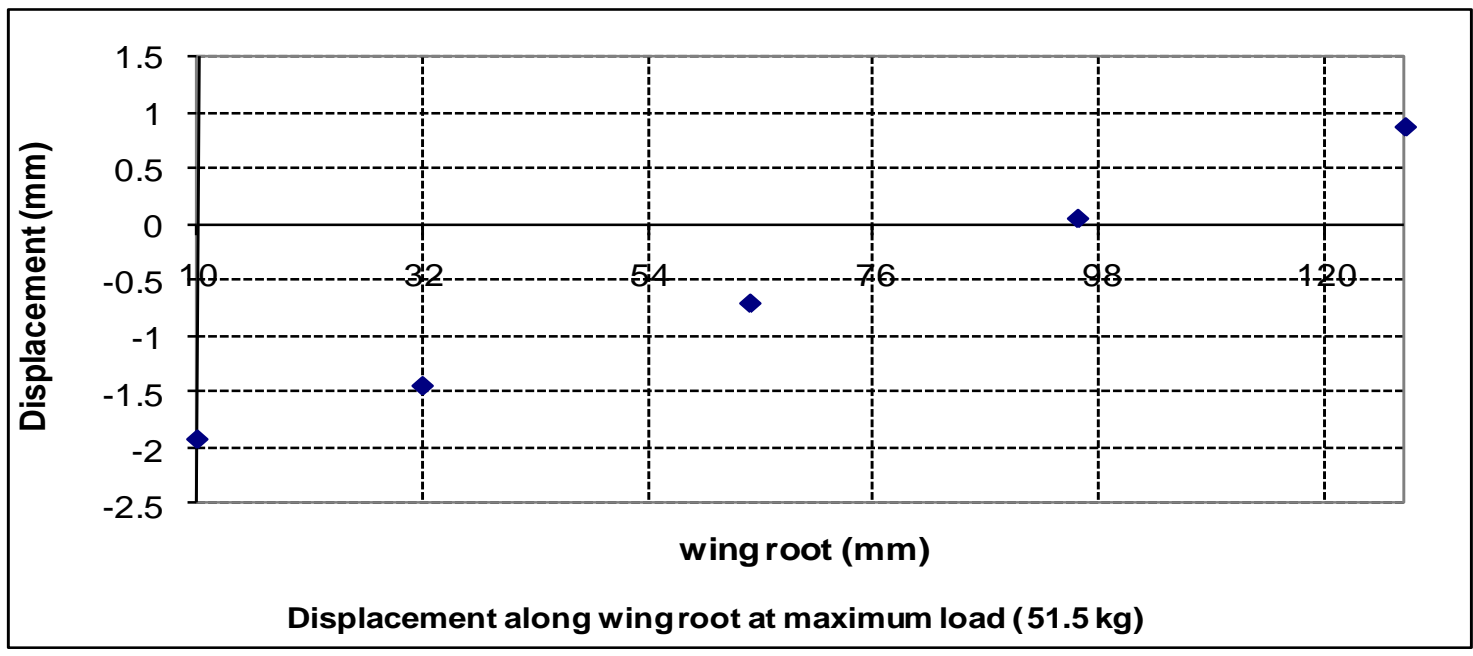

Fig.16 Displacement along the root (nodes 10, 11,12,13,14-maximum load)

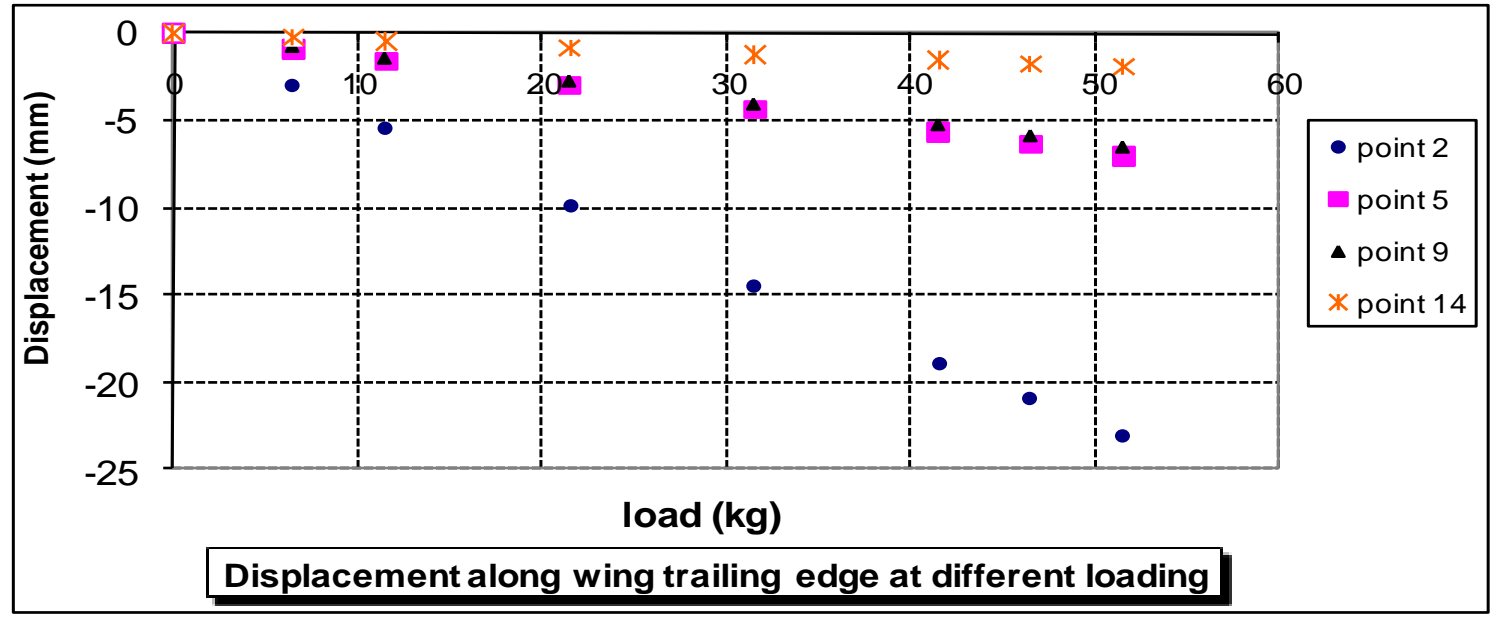

Fig. 17 Displacement along the trailing edge (nodes 2, 5, 9, and 14) 
Table 2 Displacements Measured Experimentally

$\begin{array}{rccccccccccccccc} & 1 & 2 & 3 & 4 & 5 & 6 & 7 & 8 & 9 & 10 & 11 & 12 & 13 & 14 \\ 1 & 0.4233 & 0.4578 & 0.1612 & 0.2267 & 0.1385 & 0.0128 & 0.0308 & 0.0485 & 0.1260 & -0.018 & -0.002 & 0.0136 & 0.0281 & 0.0378 \\ 2 & 0.4406 & 0.5630 & 0.1430 & 0.2489 & 0.1638 & 0.0038 & 0.0292 & 0.0551 & 0.1522 & -0.034 & -0.009 & 0.0138 & 0.0356 & 0.0503 \\ 3 & 0.1896 & 0.01873 & 0.1356 & 0.1254 & 0.0595 & 0.0219 & 0.0256 & 0.0279 & 0.0607 & 0.0076 & 0.0101 & 0.0122 & 0.0151 & 0.0166 \\ 4 & 0.2176 & 0.2628 & 0.099 & 0.1546 & 0.1003 & 0.0070 & 0.0226 & 0.0385 & 0.1031 & -0.018 & -0.002 & 0.0123 & 0.0268 & 0.0366 \\ 5 & 0.2896 & 0.3805 & 0.1034 & 0.2086 & 0.1493 & 0.0025 & 0.0280 & 0.0544 & 0.1523 & -0.034 & -0.0077 & 0.0166 & 0.0403 & 0.0565 \\ 6 & 0.0201 & 0.0092 & 0.0255 & 0.0133 & 0.0005 & 0.0187 & 0.0112 & 0.0026 & -0.0056 & 0.0205 & 0.0129 & 0.0056 & -0.0011 & -0.0058 \\ 7 & 0.0558 & 0.0600 & 0.0361 & 0.0441 & 0.0238 & 0.0133 & 0.0137 & 0.0165 & 0.0335 & 0.0064 & 0.0079 & 0.0092 & 0.0110 & 0.0122 \\ 8 & 0.0938 & 0.1208 & 0.0418 & 0.0780 & 0.0536 & 0.0026 & 0.0154 & 0.0282 & 0.08943 & -0.0167 & -0.00219 & 0.01183 & 0.02573 & 0.03559 \\ 9 & 0.1099 & 0.0152 & 0.0355 & 0.0942 & 0.0710 & -0.0064 & 0.0140 & 0.0361 & 0.1156 & -0.0345 & -0.0103 & 0.0130 & 0.0354 & 0.0516 \\ 10 & -0.0197 & -0.0388 & 0.0058 & -0.0203 & -0.0200 & 0.0147 & 0.0021 & -0.0097 & -0.0372 & 0.0710 & 0.0291 & 0.0057 & -0.0166 & -0.0345 \\ 11 & -0.0038 & -0.0114 & 0.0047 & -0.0041 & -0.0057 & 0.0087 & 0.0032 & -0.0019 & -0.0107 & 0.0337 & 0.0180 & 0.0079 & -0.0024 & -0.0110 \\ 12 & 0.0108 & 0.0121 & 0.0082 & 0.0102 & 0.0060 & 0.0041 & 0.0044 & 0.0052 & 0.0119 & 0.0069 & 0.0067 & 0.00851 & 0.00946 & 0.01066 \\ 13 & 0.0219 & 0.0322 & 0.0082 & 0.0218 & 0.0171 & -0.0015 & 0.0049 & 0.0117 & 0.0341 & -0.0163 & -0.0026 & 0.0086 & 0.02158 & 0.03387 \\ 14 & 0.0532 & 0.0707 & 0.0246 & 0.0465 & 0.0339 & -0.0017 & 0.0097 & 0.0212 & 0.0601 & -0.030 & -0.0078 & 0.0108 & 0.0336 & 0.0558\end{array}$

\begin{tabular}{|c|c|c|c|c|c|c|c|c|c|c|c|c|c|}
\hline \multirow[t]{14}{*}{2.3623} & 2.1847 & 6.2074 & 4.4119 & 7.2231 & 78.201 & 32.534 & 20.639 & 7.9366 & -55.56 & -711.2 & 73.749 & 35.659 & 26.481 \\
\hline & 1.7765 & 6.9921 & 4.0180 & 6.1055 & 274.44 & 34.295 & 18.162 & 6.5719 & -29.39 & -110.9 & 72.399 & 28.071 & 19.875 \\
\hline & & 7.4074 & 8.0117 & 16.916 & 46.306 & 39.484 & 36.092 & 16.604 & 137.10 & 101.02 & 83.045 & 67.079 & 60.798 \\
\hline & & & 6.4842 & 9.9887 & 165.28 & 44.657 & 26.056 & 9.7234 & -55.89 & -498.7 & 82.375 & 37.480 & 27.373 \\
\hline & & & & 6.7032 & 600.63 & 35.9197 & 18.4013 & 6.5741 & -29.390 & -131.53 & 60.4115 & 24.873 & 17.733 \\
\hline & & & & & 57.418 & 91.087 & 556.34 & 40.264 & 57.335 & 84.356 & 180.006 & -138.92 & -31.127 \\
\hline & & & & & & 73.410 & 60.944 & 29.998 & 157.68 & 126.906 & 109.19 & 91.546 & 82.032 \\
\hline & & & & & & & 35.502 & 11.1848 & -60.008 & -474.76 & 84.593 & 38.8813 & 28.1056 \\
\hline & Symmetric & & & & & & & 8.6612 & -28.9934 & -97.7215 & 77.4568 & 28.3145 & 19.3911 \\
\hline & & & & & & & & & 14.097 & 34.383 & 177.852 & -60.611 & -29.042 \\
\hline & & & & & & & & & & 55.620 & 126.73 & -439.32 & -91.623 \\
\hline & & & & & & & & & & & 117.483 & 105.722 & 93.7790 \\
\hline & & & & & & & & & & & & 46.3586 & 29.5315 \\
\hline & & & & & & & & & & & & & 17.959 \\
\hline
\end{tabular}

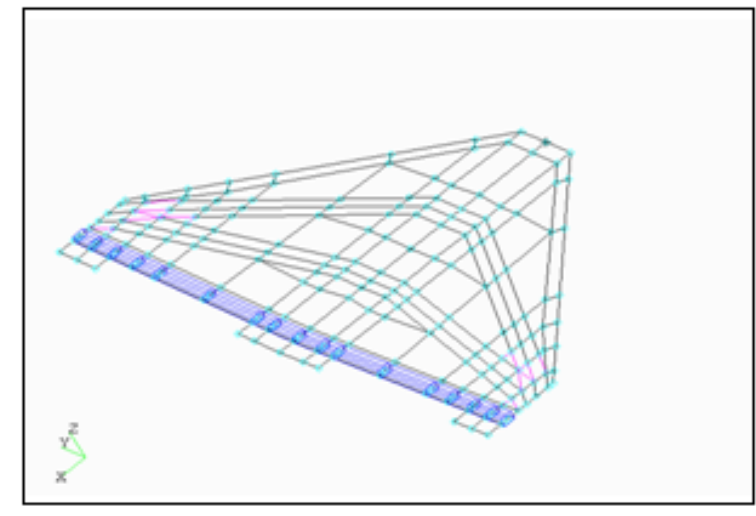

Fig 18. Composite wing model- spar location

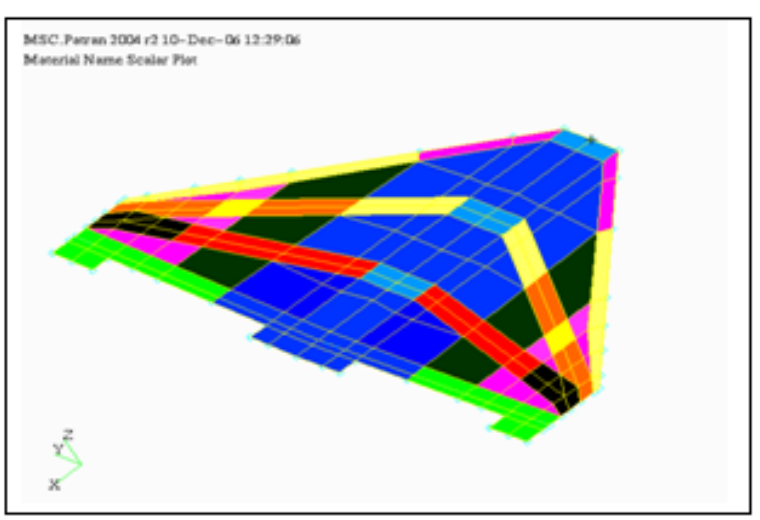

Fig19. Composite wing model 
Table 3 Material Mechanical Properties [1,2]

\begin{tabular}{l|l|l}
\hline \hline \multicolumn{1}{c|}{ Mechanical properties } & \multicolumn{1}{c}{ Symbol } & \multicolumn{1}{c}{ Value } \\
\hline Young's Modules & $\mathrm{E}_{1}, \mathrm{E}_{2}$ & $25 G N / \mathrm{m}^{2}$ \\
\hline Modulus of Rigidity & $\mathrm{G}_{12}$ & $4 G N / \mathrm{m}^{2}$ \\
\hline Poison's Ratio & $v$ & 0.26 \\
\hline Density & $\rho$ & $1.9 \mathrm{E}+3 \mathrm{~kg} / \mathrm{m}^{3}$ \\
\hline
\end{tabular}

Table 4 Displacements Calculated from Finite Element Model

$\begin{array}{rccccccccccccccc} & 1 & 2 & 3 & 4 & 5 & 6 & 7 & 8 & 9 & 10 & 11 & 12 & 13 & 14 \\ 1 & 0.6006 & 0.3323 & 0.2279 & 0.2247 & 0.2066 & 0.0884 & 0.0993 & 0.1033 & 0.0993 & 0.0041 & 0.0108 & 0.0243 & 0.0413 & 0.0456 \\ 2 & 0.3323 & 0.4713 & 0.1906 & 0.2024 & 0.2413 & 0.0638 & 0.0876 & 0.1153 & 0.1358 & -0.0184 & 0.0046 & 0.0246 & 0.0596 & 0.0835 \\ 3 & 0.2279 & 0.1906 & 0.3277 & 0.1858 & 0.1351 & 0.0966 & 0.0991 & 0.0881 & 0.0703 & 0.0158 & 0.0135 & 0.0239 & 0.0325 & 0.0268 \\ 4 & 0.2247 & 0.2024 & 0.1858 & 0.2261 & 0.1578 & 0.0740 & 0.0883 & 0.0983 & 0.0985 & -0.0022 & 0.0083 & 0.0241 & 0.0474 & 0.0576 \\ 5 & 0.2066 & 0.2413 & 0.1351 & 0.1578 & 0.2875 & 0.0431 & 0.0721 & 0.1142 & 0.1530 & -0.0286 & 0.0007 & 0.0243 & 0.0705 & 0.1058 \\ 6 & 0.0884 & 0.0638 & 0.0966 & 0.0740 & 0.0431 & 0.2158 & 0.0765 & 0.0377 & 0.0169 & 0.0486 & 0.0243 & 0.0178 & 0.0097 & -0.0040 \\ 7 & 0.0993 & 0.0876 & 0.0991 & 0.0883 & 0.0721 & 0.0765 & 0.1020 & 0.0620 & 0.0585 & 0.0107 & 0.0116 & 0.0232 & 0.0314 & 0.0319 \\ 8 & 0.1033 & 0.1153 & 0.0881 & 0.0983 & 0.1142 & 0.0377 & 0.0620 & 0.1296 & 0.1087 & -0.0191 & 0.0019 & 0.0250 & 0.0669 & 0.0906 \\ 9 & 0.0993 & 0.1358 & 0.0703 & 0.0985 & 0.1530 & 0.0169 & 0.0585 & 0.1087 & 0.2169 & -0.0404 & -0.0040 & 0.0238 & 0.0846 & 0.1367 \\ 10 & 0.0041 & -0.0184 & 0.0158 & -0.0022 & -0.0286 & 0.0486 & 0.0107 & -0.0191 & -0.0404 & 0.3712 & 0.0488 & -0.0012 & -0.0262 & -0.0531 \\ 11 & 0.0108 & 0.0046 & 0.0135 & 0.0083 & 0.0007 & 0.0243 & 0.0116 & 0.0019 & -0.0040 & 0.0488 & 0.0766 & 0.0062 & -0.0026 & -0.0090 \\ 12 & 0.0243 & 0.0246 & 0.0239 & 0.0241 & 0.0243 & 0.0178 & 0.0232 & 0.0250 & 0.0238 & -0.0012 & 0.0062 & 0.1094 & 0.0233 & 0.0223 \\ 13 & 0.0413 & 0.0596 & 0.0325 & 0.0474 & 0.0705 & 0.0097 & 0.0314 & 0.0669 & 0.0846 & -0.0262 & -0.0026 & 0.0233 & 0.1252 & 0.0935 \\ 14 & 0.0456 & 0.0835 & 0.0268 & 0.0576 & 0.1058 & -0.0040 & 0.0319 & 0.0906 & 0.1367 & -0.0531 & -0.0090 & 0.0223 & 0.0935 & 0.1804\end{array}$

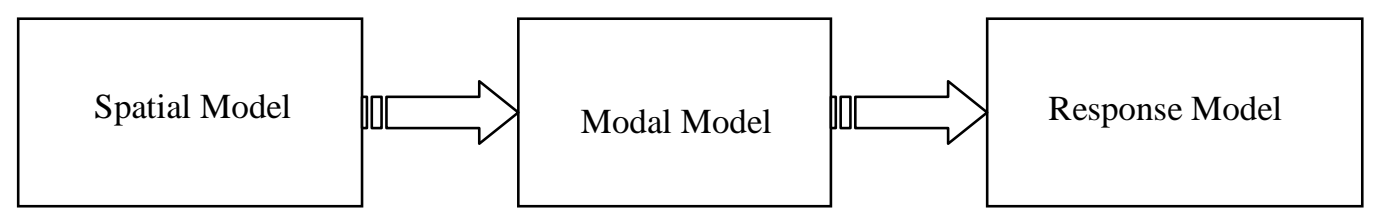

Fig. 20 Theoretical route to analysis

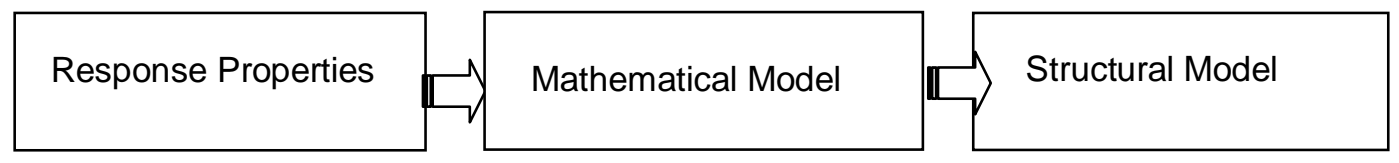

Fig. 21 Experimental route to analysis 